\title{
ON THE EXPRESSION OF A NUMBER AS THE SUM \\ OF TWO SQUARES IN TOTALLY REAL ALGEBRAIC NUMBER FIELDS ${ }^{1}$
}

\author{
WERNER SCHAAL
}

Introduction. Let $K$ be a totally real algebraic number field of degree $n$ and with discriminant $d$. Let a be an ideal of $K$ which may be integral or fractional. The number of solutions of the equation

$$
\xi=\mu^{2}+\nu^{2} \quad(\xi \in \mathfrak{a})
$$

in numbers $\mu, \nu \in \mathfrak{a}$ is denoted by $f(\xi, \mathfrak{a})$. For $x_{1}, \cdots, x_{n}$ being positive real numbers the following theorem will be proved:

THEOREM.

$$
\sum_{0<\xi^{(h)<x_{h} ; \mathfrak{a} \mid \xi}} f(\xi, \mathfrak{a})=\frac{\pi^{n}}{d N \mathfrak{a}^{2}}\left(x_{1} \cdots x_{n}\right)+R\left(x_{1}, \cdots, x_{n}\right) .
$$

(The index $h$ always takes on the values $1, \cdots, n$ if not otherwise indicated.) For any $\delta>0, x_{1} \cdots x_{n} \rightarrow \infty$, then

$$
R\left(x_{1}, \cdots, x_{n}\right)=O\left(\left(x_{1} \cdots x_{n}\right)^{n /(n+1)+\delta}\right)
$$

holds.

This result has been already proved in [4] for the case $n=2$, $\mathfrak{a}=(1)$. There was also shown that

$$
\limsup _{x_{1} x_{2} \rightarrow \infty} \frac{R\left(x_{1}, x_{2}\right)}{\left(x_{1} x_{2}\right)^{1 / 4}}>0 .
$$

For the proof of the theorem an identity given by Siegel in [5] for real quadratic number fields is generalized to totally real algebraic number fields. This identity will be applied to the problem in a similar way as it was done in [4].

1. In what follows the real numbers $c_{1}, \cdots, c_{5}$ are constants greater than 1 which only depend on the field $K$ and the ideal $\mathfrak{a}$ if not otherwise indicated. We define $S(\alpha)=\alpha^{(1)}+\cdots+\alpha^{(n)}$, $N(\alpha)=\alpha^{(1)} \cdots \alpha^{(n)}$ for numbers $\alpha \in K$. Let $r=n-1$, and let

Presented to the Society, November 23, 1963, received by the editors January 7 , 1964.

${ }^{1}$ Research was partially supported by contract DSR 7700 AF 49-638-42.

2 We introduce Hecke's characters for a number $\alpha \epsilon K$ with respect to these unit $\eta_{1}, \cdots, \eta_{r}$ 


$$
m=2 \pi i\left(\begin{array}{c}
m_{1} \\
\vdots \\
m_{r}
\end{array}\right)
$$

where $m_{1}, \cdots, m_{r}$ are rational integers.

The set of squares of all units of $K$ forms a group $G$ which may be generated by the $r$ independent units $\eta_{1}, \cdots, \eta_{r}{ }^{2}$ For this purpose let $E$ be the $r \times n$ matrix $\left(e_{p}^{(q)}\right), q=1, \cdots, r ; p=1, \cdots, n$ (see [2]), and let

$$
a=\left(\begin{array}{c}
\log \left|\alpha^{(1)}\right| \\
\vdots \\
\log \left|\dot{\alpha}^{(n)}\right|
\end{array}\right)
$$

Then following Hecke's definition we set

$$
\lambda_{m}(\alpha)=\exp \left\{m^{T} E a\right\} .
$$

If $\eta \in G$ it follows from the definition of the numbers $e_{p}^{(q)}$ that

$$
\lambda_{m}(\alpha \eta)=\lambda_{m}(\alpha) .
$$

Two numbers $\alpha, \beta \neq 0,0$ of $K$ are called "associated" if their quotient is an element of the group $G$. Otherwise $\alpha, \beta$ are called "not associated."

LEMma 1. If $x$ is a positive real number then

$$
\sum_{N(\xi) \leqq x}^{\prime} f(\xi, \mathfrak{a})=O(x)
$$

where the dash at the sign of summation indicates that the sum is to be taken over a set of not associated numbers $\xi \in \mathfrak{a}$.

Proof. For every number $\alpha$ of $K$ there exists a number $c_{1}$ and a unit $\eta \in G$ which only depends on $\alpha$ such that the following $n$ inequalities hold:

$$
c_{1}^{-1}|N(\alpha)|^{1 / n} \leqq\left|\alpha_{\eta}^{(h)}{ }^{(h)}\right| \leqq c_{1}|N(\alpha)|^{1 / n}, \quad h=1, \cdots, n,
$$

(see [6, Hilfssatz 6]). Because of

$$
f(\eta \xi, \mathfrak{a})=f(\xi, \mathfrak{a}), \quad \eta \in G
$$

we may choose the set of not associated numbers $\xi$ such that the following inequalities are satisfied: 


$$
c_{1}^{-1}(N \xi)^{1 / n} \leqq \xi^{(h)} \leqq c_{1}(N \xi)^{1 / n}, \quad h=1, \cdots, n .
$$

Whence we have

$$
\sum_{N(\xi) \leq x}^{\prime} f(\xi, \mathfrak{a}) \leqq \sum_{0<\xi^{(h)}<c_{1} x^{1 / n} ; \mathfrak{a} \mid \xi} f(\xi, \mathfrak{a}) .
$$

Since $f(\xi, \mathfrak{a})$ is the number of distinct pairs $(\mu, \nu), \mu, \nu \in \mathfrak{a}$ with $\xi=\mu^{2}+\nu^{2}$ it is sufficient to estimate the number of elements $\mu \in \mathfrak{a}$ which satisfy the inequalities $\left|\mu^{(h)}\right|<c_{2} x^{1 / 2 n}, h=1, \cdots, n$. Let $\alpha_{1}, \cdots, \alpha_{n}$ be a basis of the ideal $\mathfrak{a}$. We have to estimate the number of distinct $n$ tuples of rational integers $\left(k_{1}, \cdots, k_{n}\right)$ for which the inequalities

$$
-c_{2} x^{1 / 2 n}<\sum_{p=1}^{n} k_{p} \alpha_{p}^{(h)}<c_{2} x^{1 / 2 n}, \quad h=1, \cdots, n
$$

hold. Since $\left|\operatorname{det}\left(\alpha_{p}^{(h)}\right)\right|=N \mathfrak{a} \sqrt{ } \not \neq 0$ we obtain that there are at most $c_{3} \sqrt{ } x$ of such $n$-tuples. This proves the lemma.

For each character (1) we define the function

$$
\Phi_{m}(s, \mathfrak{a})=\sum_{\xi} \frac{f(\xi, \mathfrak{a}) \lambda_{m}(\xi)}{N(\xi)^{\mathfrak{s}}},
$$

where by $s=\sigma+i t$ a complex variable is denoted. Applying the method of partial summation it is an easy consequence of Lemma 1 that the functions $\Phi_{m}(s, a)$ converge absolutely and uniformly for $\sigma>1$.

Let $R$ be the determinant

$$
\left|\begin{array}{cccc}
1 & \log \eta_{1}^{(1)} & \cdots & \log \eta_{r}^{(1)} \\
& \vdots & \vdots \\
1 & \log \eta_{1}^{(n)} & \cdots & \cdot \log \eta_{r}^{(n)}
\end{array}\right|
$$

moreover, we introduce the abbreviation

$$
E_{p}(m)=2 \pi \sum_{q=1}^{r} m_{q} e_{p}^{(q)}, \quad p=1, \cdots, n .
$$

Then the following lemma holds:

Lemma 2. Let $x_{1}, \cdots, x_{n}$ be positive real numbers and let

$$
g\left(x_{1}, \cdots, x_{n}\right)=\sum_{0<\xi^{(h)} x_{h}<1 ; \mathfrak{a} \mid \xi} f(\xi, \mathfrak{a}) \prod_{p=1}^{n}\left(1-\xi^{(p)} x_{p}\right) .
$$

Then we have for $\sigma>1$ : 


$$
\begin{aligned}
g\left(x_{1}, \cdots x_{n}\right)=\frac{n}{2 \pi i|R|} \sum_{m_{1}, \cdots, m_{r i}-\infty}^{+\infty} \int_{\sigma-i \infty}^{\sigma+i \infty} \Phi_{m}(s, \mathfrak{a}) \\
\cdot \prod_{p=1}^{n} \frac{x_{p}^{-s+i E_{p}(m)}}{\left(s-i E_{p}(m)\right)\left(s+1-i E_{p}(m)\right)} d s .
\end{aligned}
$$

Proof. The proof of the given identity proceeds on the same lines as the proof in the case $n=2$ given in [5]. We define the column vectors

$$
k=\left(\begin{array}{c}
k_{1} \\
\vdots \\
k_{r}
\end{array}\right), \quad v=\left(\begin{array}{c}
v_{1} \\
\vdots \\
v_{r}
\end{array}\right), \quad y^{(p)}=\left(\begin{array}{c}
\log \eta_{1}^{(p)} \\
\vdots \\
\log \eta_{r}^{(p)}
\end{array}\right) \quad(p=1, \cdots, n)
$$

where $k_{1}, \cdots, k_{r}$ are rational integers and $v_{1}, \cdots, v_{r}$ are real variables. Making the substitution

$$
x_{p}=u \exp \left\{v^{T} y^{(p)}\right\}, \quad p=1, \cdots, n
$$

we observe that the function $g\left(x_{1}, \cdots, x_{n}\right)$ becomes a periodic function with respect to $v_{1}, \cdots, v_{r}$ because of property (2). The period is 1 with respect to each of the variables. Furthermore, $g\left(x_{1}, \cdots, x_{n}\right)$ is a continuous function and has piecewise continuous partial derivatives with respect to $v_{1}, \cdots, v_{r}$. Whence $g\left(x_{1}, \cdots, x_{n}\right)$ furnishes an absolutely convergent Fourier series. Denoting the right-hand side of (3) by $t^{(p)}(v)$ its coefficient is given by:

$$
\begin{aligned}
& a_{m}(u)= \int_{0}^{1} \cdots \int_{0}^{1} \exp \left\{-v^{T} m\right\} \sum_{0<\xi^{(h)} t^{(h)}(v)<1 ; \mathfrak{a} \mid \xi} f(\xi, \mathfrak{a}) \\
& \cdot \prod_{p=1}^{n}\left(1-\xi^{(p)} t^{(p)}(v)\right) d v_{1} \cdots d v_{r} \\
&= \int_{0}^{1} \cdots \int_{0}^{1} \exp \left\{-v^{T} m\right\} \sum_{0<N(\xi)<u^{-n}}^{\prime} f(\xi, \mathfrak{a}) \\
& \cdot \sum_{k_{1}, \cdots, k_{r}-\infty}^{\infty} 0<\xi^{(h)} \sum_{t^{(h)}(v+k)<1} \prod_{p=1}^{n}\left(1-\xi^{\left.(p) t^{(p)}(v+k)\right) d v_{1} \cdots d v_{r}}\right. \\
&= \int_{0}^{1} \cdots \int_{0}^{1} \exp \left\{-v^{T} m\right\} \sum_{k_{1}, \cdots, k_{r}-\infty}^{\infty} \sum_{0<N(\xi)<u^{-n}} f(\xi, \mathfrak{a}) \\
& \cdot \sum_{0<\xi^{(h)} t^{(h)}(v+k)<1} \prod_{p=1}^{n}(\cdots) d v_{1} \cdots d v_{r} .
\end{aligned}
$$


We are allowed to interchange the integration and the summation with respect to $k_{1}, \cdots, k_{r}$ because the sum is finite. Making the change of variables $v_{q}+k_{q} \rightarrow v_{q}, q=1, \cdots, r$, we obtain:

$$
\begin{aligned}
a_{m}(u)=\int_{-\infty}^{+\infty} \cdots \int_{-\infty}^{+\infty} \exp \left\{-v^{T} m\right\} & \sum_{0<\xi^{(h)} t^{(h)}(v)<1}^{\prime} f(\xi, \mathfrak{a}) \\
& \cdot \prod_{p=1}^{n}\left(1-\xi^{(p)} t^{(p)}(v)\right) d v_{1} \cdots d v_{r} .
\end{aligned}
$$

Now we form the integral $\int_{0}^{\infty} u^{n \varepsilon-1} a_{m}(u) d u$ for $\sigma>1$. Making the change of variables (3) we get:

$$
\begin{aligned}
\int_{0}^{\infty} u^{n s-1} a_{m}(u) d u & \\
= & \frac{1}{|R|} \int_{0}^{\infty} \cdots \int_{0}^{\infty} \prod_{p=1}^{n} x_{p}^{s-1-i E_{p}(m)} \sum_{0<\xi^{(h)} x_{h}<1}^{\prime} f(\xi, \mathfrak{a}) \\
& =\frac{1}{|R|} \prod_{\xi=1}^{n}\left(1-\xi^{(p)} x_{p}\right) d x_{1} \cdots d x_{n} \\
& =\frac{1}{|R|} \Phi_{m}(s, \mathfrak{a}) \prod_{p=1}^{n}\left[\left(s-i E_{p}(m)\right)\left(s+1-i E_{p}(m)\right)\right]^{-1} .
\end{aligned}
$$

The application of Mellin's inversion formula yields for $\sigma>1$ :

$a_{m}(u)=\frac{n}{2 \pi i|R|} \int_{\sigma-i \infty}^{\sigma+i \infty} u^{-n s} \Phi_{m}(s, \mathfrak{a}) \prod_{p=1}^{n}\left[\left(s-i E_{p}(m)\right)\left(s+1-i E_{p}(m)\right)\right]^{-1} d s$.

Since

$$
g\left(x_{1}, \cdots, x_{n}\right)=\sum_{m_{1}, \cdots, m_{r=-\infty}}^{\infty} a_{m}(u) \exp \left\{v^{T} m\right\},
$$

this proves the lemma.

Let

$$
F\left(v_{1}, \cdots, v_{n}\right)=\sum_{0<\xi^{(h)}<v_{h}} f(\xi, \mathfrak{a}) .
$$

Then we have 


$$
\begin{aligned}
\left(x_{1} \cdots x_{n}\right) g\left(\frac{1}{x_{1}}, \cdots,\right. & \left.\frac{1}{x_{n}}\right) \\
& =\sum_{0<\xi^{(h)<x_{h} ; a \mid \xi}} f(\xi, \mathfrak{a}) \prod_{p=1}^{n}\left(x_{p}-\xi^{(p)}\right) \\
& =\sum_{0<\xi^{(h)<x_{h} ; \mathfrak{a} \mid \xi}} \int_{\xi^{(1)}}^{x_{1}} \cdots \int_{\xi^{n}}^{x_{n}} f(\xi, \mathfrak{a}) d v_{1} \cdots d v_{n} \\
& =\int_{0}^{x_{1}} \cdots \int_{0}^{x_{n}} F\left(v_{1}, \cdots, v_{n}\right) d v_{1} \cdots d v_{n} .
\end{aligned}
$$

An elementary calculation furnishes the result:

$$
\begin{aligned}
\int_{0}^{y_{1}} \cdots \int_{0}^{y_{n}} F\left(x_{1}\right. & \left.+v_{1}, \cdots, x_{n}+v_{n}\right) d v_{1} \cdots d v_{n} \\
& =\frac{n}{2 \pi i|R|} \sum_{m_{1}, \cdots, m_{r}=-\infty}^{+\infty} \int_{\sigma-i \infty ; \sigma>1}^{\sigma+i \infty} \\
& \cdot \prod_{p=1}^{n} \frac{\left(y_{p}+x_{p}\right)^{s+1-i E_{p}(m)}-x_{p}^{s+1-i E_{p}(m)}}{\left(s-i E_{p}(m)\right)\left(s+1-i E_{p}(m)\right)} \Phi_{m}(s, \mathfrak{a}) d s .
\end{aligned}
$$

2. The left-hand side of (4) may be abbreviated by $J$. Since $f(\xi, \mathfrak{a}) \geqq 0$ we obtain the inequality:

$$
F\left(x_{1}, \cdots, x_{n}\right) \leqq\left(y_{1} \cdots y_{n}\right)^{-1} J \leqq F\left(x_{1}+y_{1}, \cdots, x_{n}+y_{n}\right) .
$$

We observe from this inequality that the asymptotic behaviours of $F\left(x_{1}, \cdots, x_{n}\right)$ and $\left(y_{1} \cdots y_{n}\right)^{-1} J$ are the same. Therefore we shall try to find an approximation of $J$. For this purpose the functions $\Phi_{m}(s, \mathfrak{a})$ are analytically continued over the whole $s$-plane. Let:

$$
\Theta\left(z_{1}, \cdots, z_{n} ; \mathfrak{a}\right)=\sum_{\mathfrak{a} \mid \mu} \exp \left\{-\frac{\pi}{\sqrt[n]{ }\left(d N \mathfrak{a}^{2}\right)} \sum_{p=1}^{n} \mu^{(p) 2} z_{p}\right\},
$$

$z_{1}, \cdots, z_{n}$ being complex variables with $\operatorname{Re} z_{h}>0, h=1, \cdots, n$; then Hecke proved in [3]:

$$
\Theta\left(z_{1}, \cdots, z_{n} ; \mathfrak{a}\right)=\left(z_{1} \cdots z_{n}\right)^{-1 / 2} \Theta\left(\frac{1}{z_{1}}, \ldots, \frac{1}{z_{n}} ; \frac{1}{\mathfrak{a d}}\right),
$$

where $\delta$ is the ramification ideal of the field $K$. Well known calculations and the application of (5) lead to the equation: 


$$
\begin{aligned}
& \left(\frac{d N \mathfrak{a}^{2}}{\pi^{n}}\right)^{s} \frac{1}{|R|} \Phi_{m}(s, \mathfrak{a}) \prod_{p=1}^{n} \Gamma\left(s-i E_{p}(m)\right) \\
& =\frac{b_{m}}{s(s-1)}+\int_{u=1}^{u=\infty} \int_{-1 / 2}^{1 / 2} \cdots \int_{-1 / 2}^{1 / 2}\left[\Theta ^ { 2 } \left(u \eta_{1}^{(1) v_{1}} \cdots \eta_{r}^{(1) v_{r}}, \cdots,\right.\right. \\
& \left.+\int_{u=1}^{u=\infty} \int_{-1 / 2}^{1 / 2} \cdots \int_{-1 / 2}^{1 / 2}\left[\Theta_{1}^{(n) v_{1}} \cdots \eta_{r}^{(n) v_{r}} ; \mathfrak{a}\right)-1\right] u^{n s} \exp \left\{-v^{T} m\right\} d v_{1}{ }^{(1) v_{1}} \cdots d v_{r} \frac{d u}{u} \\
& \eta_{\eta_{r}}^{(1) v_{r}}, \cdots, \\
& \left.\left.{ }^{(n) v_{1}} \cdots \eta_{r}{ }^{(n) v_{r}} ; \frac{1}{\mathfrak{a} \mathfrak{d}}\right)-1\right] u^{n(1-s)} \exp \left\{v^{T} m\right\} d v_{1} \cdots d v_{r} \frac{d u}{u}
\end{aligned}
$$

with

$$
b_{m}= \begin{cases}1 / n & \text { if } m_{1}=\cdots=m_{r}=0, \\ 0 & \text { otherwise. }\end{cases}
$$

If $m_{1}^{2}+\cdots+m_{r}^{2}>0$ the right-hand side of (6) is an integral function of $s$; if $m_{1}=\cdots=m_{r}=0$ there are two simple poles at $s=0$ and $s=1$. So we recognize that $\Phi_{m}(s, \mathfrak{a})$ is an integral function of $s$ except in the case $m_{1}=\cdots=m_{r}=0 ; \Phi_{0}(s, \mathfrak{a})$ has a simple pole at $s=1$. Another immediate consequence of equation (6) is the functional equation

(7) $\Phi_{m}(s, \mathfrak{a})=\left(\frac{d N \mathfrak{a}^{2}}{\pi^{n}}\right)^{1-2 s} \prod_{p=1}^{n} \frac{\Gamma\left(1-s+i E_{p}(m)\right)}{\Gamma\left(s-i E_{p}(m)\right)} \Phi_{-m}\left(1-s, \frac{1}{\mathfrak{a} \mathfrak{d}}\right)$,

which holds for all $m_{1}, \cdots, m_{r}$.

By equations (6) and (7) we can estimate the functions $\Phi_{m}(s, \mathfrak{a})$ uniformly in $m_{1}, \cdots, m_{r}$ in the infinite strip $-\epsilon \leqq \sigma \leqq 1+\epsilon, \epsilon>0$. If we apply Phragmén-Lindelöf's extension of the maximum-modulus theorem to the functions $\Phi_{m}(s, \mathfrak{a})$ we obtain the inequalities:

$$
\begin{aligned}
\left|\Phi_{m}(\sigma+i t, \mathfrak{a})\right| \leqq c_{4}(\epsilon) & \prod_{p=1}^{n}\left(1+\left|t-E_{p}(m)\right|\right)^{1-\sigma+\epsilon}, \\
& -\epsilon \leqq \sigma \leqq 1+\epsilon, m_{1}^{2}+\cdots+m_{r}^{2}>0 .
\end{aligned}
$$

Inequality (8) also holds for $\Phi_{0}(s, \mathfrak{a})$ if $|t| \geqq c_{5}$. (The calculations which lead to (8) are given very explicitly for a similar case in [1].)

3. Now it is easy to investigate the asymptotic behaviour of the right-hand side of $(4)$ for $\left(x_{1} \cdots x_{n}\right) \rightarrow \infty$. The path of integration in (4) is replaced by a straight line in the critical strip whose point of 
intersection with the real axis may be $\sigma=\delta, 0<\delta<1$. Considering the pole of $\Phi_{0}(s, \mathfrak{a})$ at $s=1$ we find:

$$
J=\frac{1}{2^{n}} \frac{\pi^{n}}{d N a^{2}} \prod_{p=1}^{n}\left[\left(y_{p}+x_{p}\right)^{2}-x_{p}^{2}\right]
$$

$$
\begin{aligned}
&+\frac{n}{2 \pi i|R|} \sum_{m_{1}, \cdots, m_{r}--\infty}^{+\infty} \int_{\delta-i \infty}^{\delta+i \infty} \Phi_{m}(s, \mathfrak{a}) \\
& \cdot \prod_{p=1}^{n} \frac{\left(y_{p}+x_{p}\right)^{o+1-i E_{p}(m)}-x_{p}^{\bullet+1-i E_{p}(m)}}{\left(s-i E_{p}(m)\right)\left(s+1-i E_{p}(m)\right)} d s, \\
& s=\delta+i t, 0<\delta<1 .
\end{aligned}
$$

The infinite sum in (9) can be easily estimated if one considers that the following determinant does not vanish for $1 \leqq k \leqq n$ :

$$
\left|\begin{array}{cccc}
e_{k}^{(1)}-e_{1}^{(1)} \cdots e_{k}^{(1)}-e_{k-1}^{(1)} & e_{k}^{(1)}-e_{k+1}^{(1)} \cdots e_{k}^{(1)}-e_{n}^{(1)} \\
\vdots & \vdots & \vdots & \vdots \\
\vdots & \vdots & \vdots & \vdots \\
e_{k}^{(r)}-e_{1}^{(r)} \cdots e_{k}^{(r)}-e_{k-1}^{(r)} & e_{k}^{(r)}-e_{k+1}^{(r)} \cdots e_{k}^{(r)}-e_{n}^{(r)}
\end{array}\right|
$$

Then we obtain from (9)

(10) $J=\frac{1}{2^{n}} \frac{\pi^{n}}{d N \mathfrak{a}^{2}} \prod_{p=1}^{n}\left[\left(y_{p}+x_{p}\right)^{2}-x_{p}^{2}\right]+O\left(\prod_{p=1}^{n}\left(y_{p}+x_{p}\right)^{\delta+1}\right)$.

If we choose

$$
y_{p}=x_{p}\left(x_{1} \cdots x_{n}\right)^{-1 /(n+1)}, \quad p=1, \cdots, n
$$

and divide $J$ by the product $y_{1} \cdots y_{n}$ equation (10) yields for $x_{1} \cdots x_{n} \rightarrow \infty$ and any $\delta>0$

$$
\frac{J}{y_{1} \cdots y_{n}}=\left(\frac{\pi^{n}}{d N \mathfrak{a}^{2}}\right)\left(x_{1} \cdots x_{n}\right)+O\left(\left(x_{1} \cdots x_{n}\right)^{n /(n+1)+\delta}\right) .
$$

Recalling the remark in the beginning of $\$ 2$ we observe that (11) also gives the asymptotic behaviour of $F\left(x_{1}, \cdots, x_{n}\right)$ for $x_{1} \cdots x_{n} \rightarrow \infty$ and any $\delta>0$ :

$$
F\left(x_{1}, \cdots, x_{n}\right)=\left(\frac{\pi^{n}}{d N \mathfrak{a}^{2}}\right)\left(x_{1} \cdots x_{n}\right)+O\left(\left(x_{1} \cdots x_{n}\right)^{n /(n+1)+\delta}\right) .
$$

This proves the theorem formulated in the introduction. 


\section{REFERENCES}

1. Heinrich Behnke, Analytische Funktionen und algebraische Zahlen, Abh. Math. Sem. Univ. Hamburg, 2 (1923), 81-111.

2. E. Hecke, Eine neue Art von Zetafunktionen und ihre Beziehungen zur Verteilung der Primzahlen, Math. Z., 1 (1918), 357-376.

3. - Über die Zetafunktionen beliebiger algebraischer Zahlkörper, Nachr. König. Ges. Wiss. Göttingen Math.-Phys. Kl. (1917), 77-89.

4. W. Schaal, Übertragung des Kreisproblems auf reell-quadratische Zahlkörper, Math. Ann., 145 (1962), 273-284.

5. C. L. Siegel, Mittelwerte arithmetischer Funktionen in Zahlkörpern, Trans. Amer. Math. Soc. 39 (1936), 219-224.

6. —-, Additive Theorie der Zahlkörper. II, Math. Ann. 88 (1923), 184-210.

Massachusetts Institute of Technology 